\title{
STRUCTURE AND FALLING MOTION OF EARLY SNOWFLAKES
}

\author{
by
}

\author{
MASAHIRO KAJIKAWA
}

Department of Earth Science, Akita University, Akita 010, Japan

\section{ABSTRACT}

Relations between the structure and falling motion of early snowflakes, ie those composed of a few crystals, were investigated to clarify their growth process.

The following results were obtained: (1) Two crystals of the same shape in a snowflake are similar in size, but those of different shape are considerably different in size. (2) In snowflakes having two crystals of the same shape, one crystal is attached near the center of the other; where the two are of different shapes, a smaller crystal is attached near the tip of the other. (3) The nondimensional amplitude of the spiral falling motion of snowflakes composed of two crystals of different shape, is slightly larger than for those with crystals of the same shape. This suggests that snowflakes composed of different shapes are more likely to aggregate to other snowflakes or crystals. (4) In snowflakes consisting of three crystals, the centers are usually arranged in a straight line. (5) The nondimensional amplitude of the spiral motion of snowflakes consisting of three crystals increases with departures from the straight-line arrangement of their centers.

\section{INTRODUCTION}

As a basic study for the growth process of snowflakes, it is important to analyze the structure and falling motion of early snowflakes, ie those composed of a few crystals. Magono and Oguchi (1955) observed the combined state of crystals in snowflakes and found that branches of dendritic crystals play a leading role in their aggregation. Higuchi $(1955,1960)$ observed initial growth stages of snowflakes and calculated the probability of aggregation between two crystals of the plane type. Recently, Kajikawa (1982) observed free-fall pattern of early snowflakes by a stereophotogrammetric method. He found that about $80 \%$ of snowflakes fall with spiral or rotational motion.

The purpose of this study is to analyze relations between the structure and the falling motion of early snowflakes based on previous observation (Kajikawa 1982). The data used in this analysis were obtained at Mt Teine observatory (1024 $\mathrm{m}$ a.s.l.) of Hokkaido University, during periods of continuous snowfall. Large snowflakes were not involved.

\section{SNOWFLAKES CONSISTING OF TWO CRYSTALS}

To analyze snowflakes consisting of two crystals of the plane type, crystals were divided into seven groups as shown in Table 1. For each group, an experimental formula of falling velocity vs size of crystals is available (Kajikawa 1975).

Relationship between the sizes of two component crystals of the dendritic type is shown in Figure 1. It seems likely that two crystals of the same shape are similar to each other in size because of the aggregation of two crystals in their initial stage (Nakaya 1954, Higuchi 1955). In the case of different shapes of the dendritic type, the two crystals in each pair are different in size. The characteristics mentioned above also appeared in other groups.

The combined state of component crystals is an important factor affecting falling motion, and hence the aggregation of other snowflakes or crystals. A measure (S) of the combined state of two crystals has been
TABLE 1. SEVEN GROUPS OF THE PLANE SNOW CRYSTALS CLASSIFIED AFTER THE MANNER OF MAGONO AND LEE (1966). N IS THE OBSERVED NUMBER OF SNOWFLAKES, WHICH WERE SHAPE OF TWO CRYSTALS OF THE SAME SHAPE.

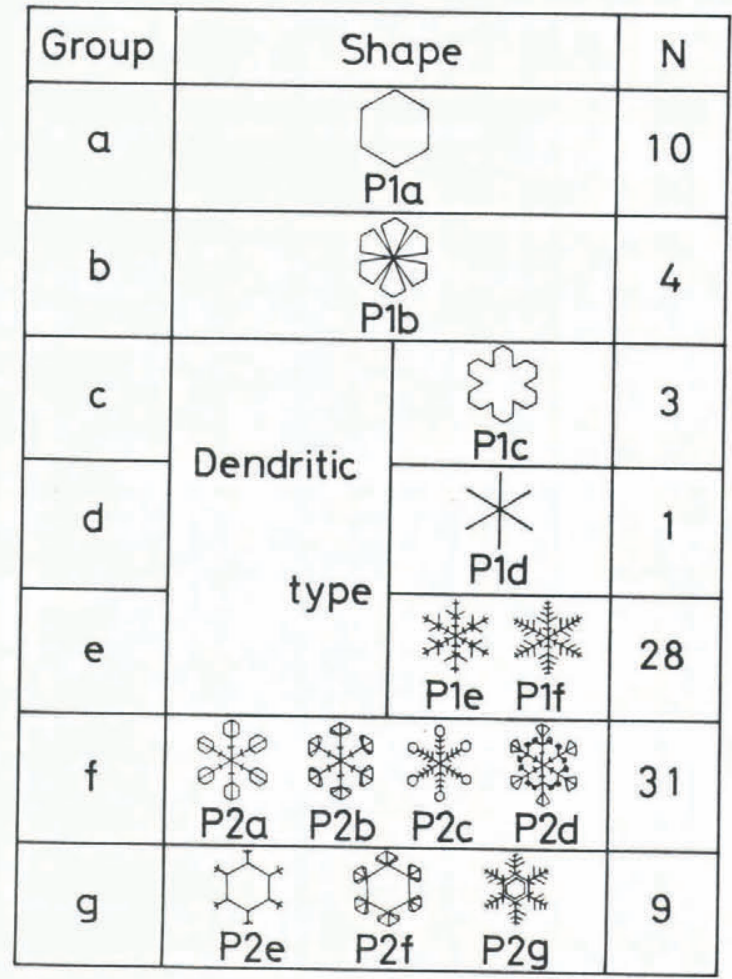

introduced by Higuchi (1960) (Figure 2), where $d_{1}$ and $d_{2}$ are the sizes of two crystals, and $l$ is the distance between their centers.

Figure 3 shows the frequency distribution of $S$ in snowflakes consisting of plane-type crystals. The distribution of $\mathrm{S}$ for the different shapes is similar to that of Higuchi's result, although its peak (about 0.65 in S) is larger than his. This means that a smaller crystal attaches near the tips of other crystal because of the large difference in their falling velocities (Kajikawa 1975). On the other hand, in crystals of the same shape, $\mathrm{S}$ has a primary peak at about 0.2 , with a secondary peak at about 0.65 . This suggests that one crystal attaches near the center of another crystal because of the small difference in their falling velocities.

Kajikawa (1982) found that $70 \%$ snowflakes consisting of two crystals show spiral or rotational falling. The relationship between the nondimensional amplitude $\left(a^{\prime}=a / d\right)$ of the spiral motion and $S$ is shown in Figure 4, where $a$ is the amplitude of the spiral path, $d$ the size of the snowflakes. It can be seen that a' for differently shaped crystals is slightly larger than that for similar crystals. Moreover, a' of the 


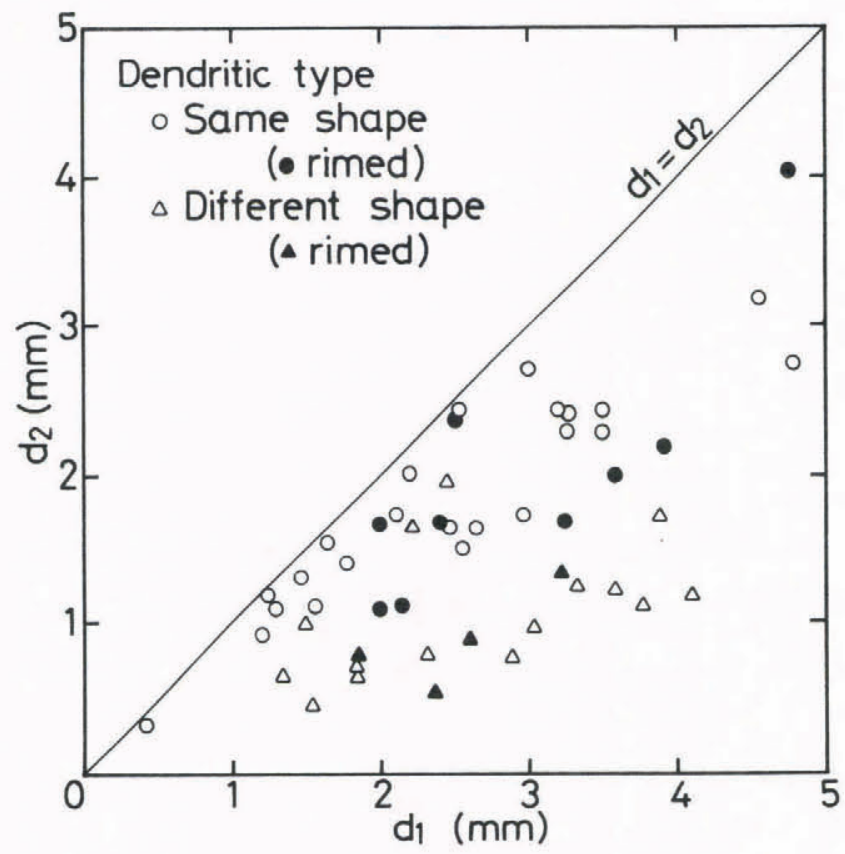

Fig.1. Relationship between the sizes $\left(d_{1}>d_{2}\right)$ of two component crystals.

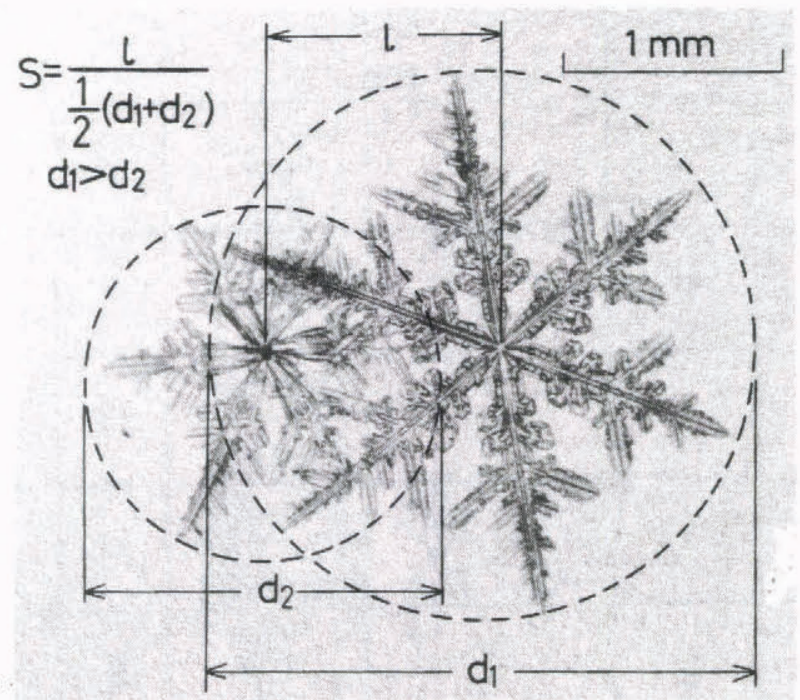

Fig.2. An example of snowflakes consisting of two dendritic crystals.

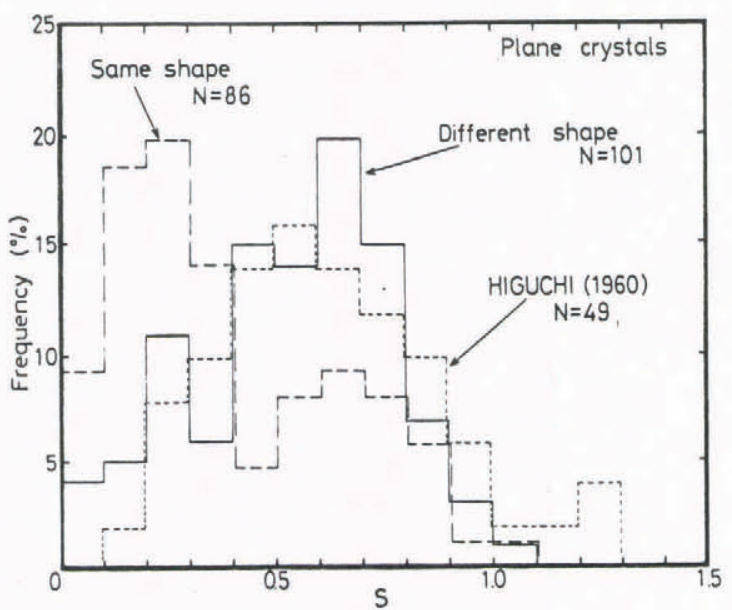

Fig.3. Frequency distribution of $\mathrm{S}$ in the snowflakes consisting of plane crystals.

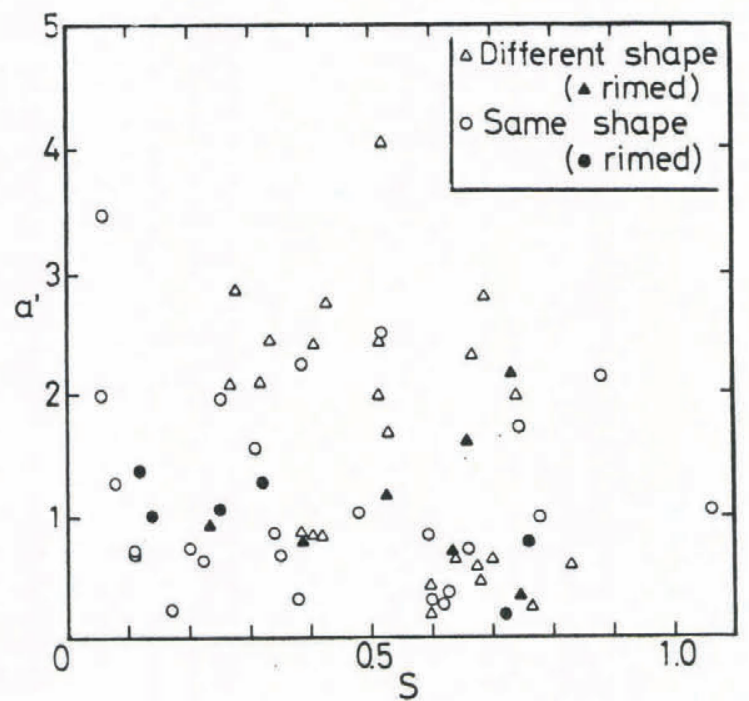

Fig.4. Relationship between the nondimensional amplitude (a') of the spiral motion and $\mathrm{S}$.

same shape is smaller than 2 , and hence the period of spiral motion is shorter than 1 second (Kajikawa 1982). These facts suggest that snowflakes consisting of different shapes are more likely to aggregate than other snowflakes or crystals, because of the increased chance of collision due to the larger amplitude of spiral or horizontal motion.

\section{SNOWFLAKES CONSISTING OF THREE CRYSTALS}

To analyze the combined state of three component crystals, a parameter $\left(A / A_{\max }\right)$ was defined as shown in Figures 5 and 6 . Here, $\mathrm{A}$ is the area of a triangle surrounded by three centers $\left(C_{1}, C_{2}\right.$ and $\left.C_{3}\right)$ of crystals, and $A_{m x}$ is the maximum value of $A$. From the frequency distribution of $A / A_{m a x}$, it is clear that most of $A / A_{\text {rax }}$ is very small. This means that there is a tendency for the centers of three crystals to be arranged in a straight line.

The relationship between the nondimensional amplitude $\left(\mathrm{a}^{\prime}=\mathrm{a} / \mathrm{d}\right)$ of the spiral falling motion and $A / A_{\max }$ is shown in Figure 7. Although the data are scattered, it can be seen that $a^{\prime}$ increases with a shift from the straight-line arrangement of the three crystals. Most of $a^{\prime}$ is smaller than about 2 and not related to the shapes of component crystals.

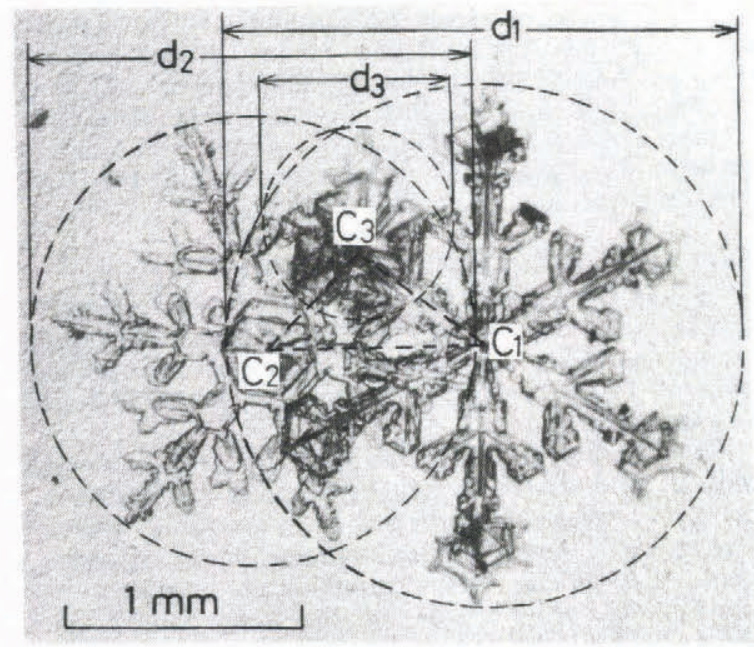

$$
\begin{aligned}
& A=\text { Area } C_{1} C_{2} C_{3} \\
& A_{\text {max }}=\frac{1}{8}\left(d_{1}+d_{2}\right)\left(d_{1}+d_{3}\right)
\end{aligned}
$$

Fig.5. An example of snowflakes consisting of three crystals. 


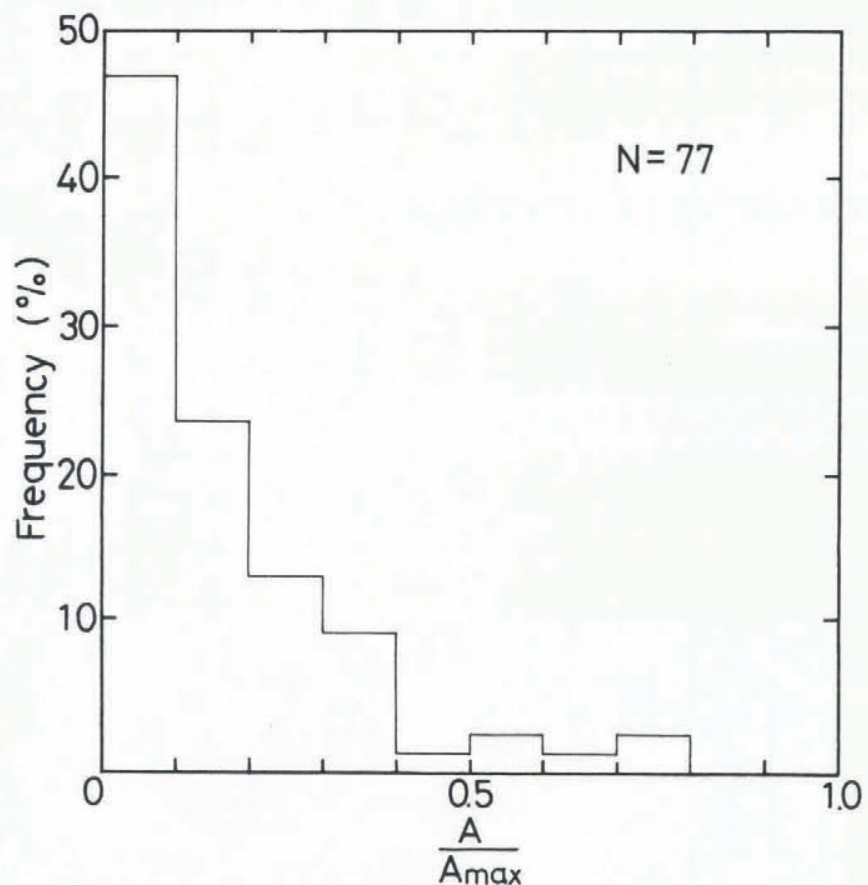

Fig.6. Frequency distribution of $A / A_{\max ^{2}}$

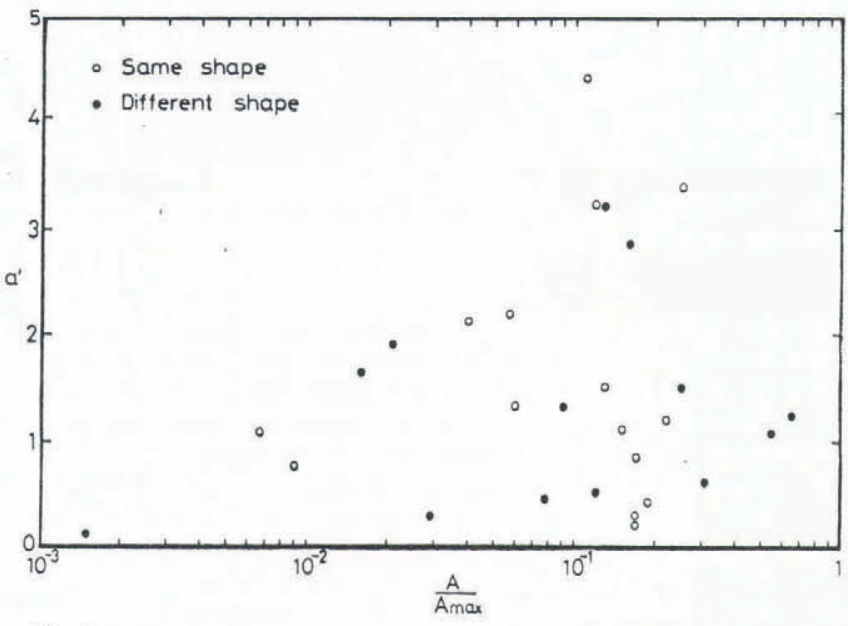

Fig.7. Relationship between the nondimensional amplitude (a') of the spiral motion and $\mathrm{A} / \mathrm{A}_{\max }$.

\section{CONCLUSIONS}

Frequency distribution of $S$ (see Figure 2) in snowflakes of the same shape shows a peak at about 0.2 . This means that one crystal becomes attached near the center of another crystal because of small difference in their falling velocities. On the other hand, $S$ of a different shape shows a peak at about 0.65 , which implies that a smaller crystal becomes attached near the tips of other crystal because of the large difference in their falling velocities.

The nondimensional amplitude of the spiral falling motion in snowflakes consisting of two crystals of different shape is slightly larger than those having crystals of the same shape. This fact suggests that snowflakes consisting of different shapes have an advantage for aggregation over other snowflakes or crystals of the same shape.

In the snowflakes consisting of three crystals, it is probable their centers will be arranged in a straight line. Nondimensional amplitude of the spiral falling motion increases with a shift from the straight-line arrangement of their centers.

\section{REFERENCES}

Higuchi K 1955 On the size of single snow crystals and of element forming snow flakes. Journal of the Meteorological Society of Japan 33(1): 9-22

Higuchi K 1960 On the coalescence between plane snow crystals. Journal of Meteorology 17(3): 239-243

Kajikawa M 1975 Experimental formula of falling velocity of snow crystals. Journal of the Meteorological Society of Japan 53(4): 267-275

Kajikawa M 1982 Observation of the falling motion of early snow flakes, Part I. Journal of the Meteorological Society of Japan 60(2): 797-803

Magono C, Oguchi H 1951 Classification of snow flakes and their structures. Journal of the Meteorological Society of Japan 33(2): 56-67

Magono C, Lee C W 1966 Meteorological classification of natural snow crystals. Journal of the Faculty of Science, Hokkaido University Ser VII 2: 321-335

Nakaya U 1954 Snow crystals. Boston, Harvard University Press 